\title{
Feature Extraction for Medical CT Images of Sports Tear Injury
}

\author{
Qi Nie ${ }^{1} \cdot$ Ye-bing Zou ${ }^{1} \cdot$ Jerry Chun-Wei Lin ${ }^{2}$ (I) \\ Accepted: 20 October 2020 / Published online: 28 October 2020 \\ (C) The Author(s) 2020
}

\begin{abstract}
Analysis of medical CT images directly affects the accuracy of clinical case diagnosis. Therefore, feature extraction problem of medical CT images is extremely important. A feature extraction algorithm for medical CT images of sports tear injury is proposed. First, CT images are decomposed into a low frequency component and a series of high frequency components in different directions by wavelet fast decomposition method. The high- and low-frequency information of CT images is enhanced by wavelet layered multi-directional image enhancement algorithm, and the multi-scale enhancement for medical CT images of sports tear injury is completed. Then, edge of the enhanced CT images is extracted using an image edge extraction algorithm based on extended mathematical morphology. Finally, based on the extracted edge information of CT images, feature extraction for medical CT images of sports tear injury is completed by the NSCT-GLCM based CT image feature extraction algorithm. Research results show that the proposed algorithm effectively extracts CT image features of sports tear injury and provides auxiliary information for doctor diagnosis.
\end{abstract}

Keywords Sports tear injury $\cdot$ Medical CT $\cdot$ Image feature $\cdot$ Extraction algorithm $\cdot$ Fast wavelet transform $\cdot$ Multi-scale enhancement

\section{Introduction}

The first step in diagnosis and treatment of a patient is to obtain enough information about the patient's condition [1]. Invention of the microscope is a major advancement in the development of medicine. Because it allows people to observe microscopic worlds that are previously invisible to naked eye in the form of images [2]. Medical application of X-rays allows people to observe the internal structure of human body and provide important information for doctors to diagnose the cause of diseases [3]. Computed tomography provides accurate structural information within human body and evolves from $2 \mathrm{D}$ to $3 \mathrm{D}$. On the basis of X-ray CT, many computed tomography techniques, such as magnetic resonance tomography, positron emission tomography, and electrical impedance tomography, have been developed. Based on these existing imaging technologies, new imaging methods are constantly being developed [4]. The first

Jerry Chun-Wei Lin

jerrylin@ieee.org

1 Physical Education Department, Jiangxi University of Traditional Chinese Medicine, Nanchan 330004, China

2 Department of Computer Science, Electrical Engineering and Mathematical Sciences, Western Norway University of Applied Sciences, 5063 Bergen, Norway step in medical image imaging is to use some kind of energy to pass through the body and measure energy after interacting with the body. Mathematical methods are then used to estimate the two-dimensional and three-dimensional distribution of this energy interacting with human tissue (absorption, attenuation, nuclear magnetic perturbations, etc.) and produce images [1]. Xray computed tomography $(\mathrm{CT})$ is based on a large number of $\mathrm{X}$-ray projection data, using mathematical reconstruction methods to obtain two-dimensional or three-dimensional X-ray density (tissue attenuation coefficient) distribution image. In recent years, due to the continuous updating and improvement of CT devices, imaging software and scanning technology, CT movies, $\mathrm{C}$ angiography, ultra-high-speed $\mathrm{CT}$, high-resolution $\mathrm{CT}$, spiral $\mathrm{CT}$ and other technologies have emerged. It enables CT technology to be applied in many areas of the clinic, improving accuracy and credibility of diagnosis [5].

With the maturity of various imaging inspection techniques, hospitals have collected a large number of $\mathrm{CT}$ images. How to mine these images and help doctors diagnose cases is of great significance [6]. Image segmentation is the basis for disease diagnosis using CT images. Therefore, improving the accuracy of CT image segmentation has become an important research topic [4]. Medical CT images are characterized by low contrast, variability in tissue properties, and ambiguity in the boundaries between soft tissues or soft tissues and disorders, as well as complexity of shape structures and distribution 
of fine structures. It creates great difficulties in studying the edges of medical images [7]. Moreover, in the past, medical CT image segmentation and feature extraction methods are mostly based on the lung and the head, and there are few studies on sports tear injure. In this paper, feature extraction of medical CT images is studied in depth, and a feature extraction algorithm for medical CT images of sports tear injury is proposed in order to extract their features with high efficiency and precision.

\section{Materials and methods}

Medical CT images of sports tear injury are an important means of information visualization [8]. They visually provide the physician with information to aid in diagnosis and treatment. In particular, experienced radiologists and clinicians get a lot of useful information from these images [9]. However, original images from scanning devices may be affected by various factors such as imaging devices and acquisition conditions, and image quality degradation or even artifacts may occur. Even with high quality images, in most cases, it is difficult to draw useful diagnoses directly with naked eye. People with different abilities and backgrounds often get different results for the same medical CT images. It is even more impossible to have a quantitative assessment of images. Therefore, the first task is to preprocess the acquired medical CT images. That is, filtering out image noise and interference, highlighting areas or edges of interested objects to lay the groundwork for further analysis [10].

\subsection{Pretreatment for CT images of sports tear injury}

\subsubsection{Wavelet decomposition for medical CT images of sports tear injury}

Wavelet decomposition is equivalent to a set of multiresolution band-pass filters. With this feature, medical CT images of sports tear injure are decomposed into sub-images in different directions. Different methods are used to study the sub-image information in different directions under its corresponding scale. Medical CT images are processed using twodimensional wavelet decomposition in this section. Medical CT image signals are filtered and extracted using two sets of filters $g(n)$ and $h(n)$. The original image is broken down into four sub-band images, denoted as $L_{1}, L_{2}, L_{3}$, and $L_{4}$, as shown in Fig. 1.

As shown in Fig. 1, two sets of filters $g(n)$ and $h(n)$ are set, use two sets of filters to filter and extract medical CT image signals, and the original image is decomposed into four subband images, which are $L_{1}, L_{2}, L_{3}$, and $L_{4}$. Let $\varphi$ and $\Phi$ be the corresponding scale function and wavelet function, respectively. For a medical CT image $f(x, y)$ of sports tear injure,

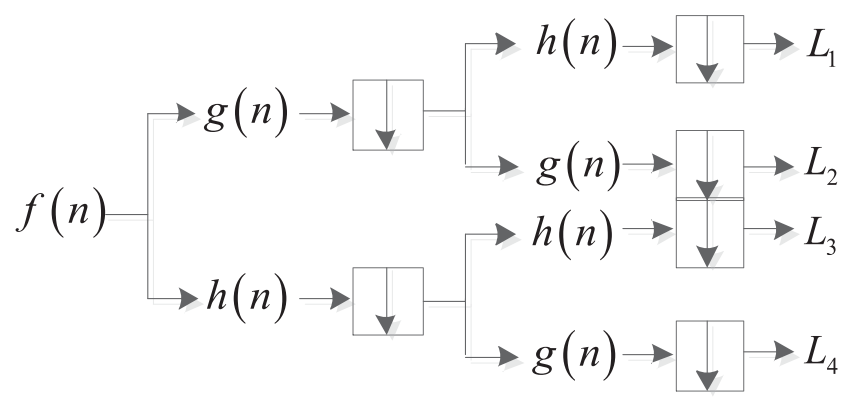

Fig. 1 Two-dimensional wavelet decomposition structure diagram

the rapid decomposition of Mallet wavelet can be expressed as:

$$
\begin{aligned}
& f(x, y)=\sum_{k, m} A_{j, k, m} g(n)+\sum_{c=h, v, d} \sum_{k, m} D_{j, k, m}^{h} h(n) \\
& A_{j, k, m}=\sum_{l, n} h_{l-2 k} h_{n-2 m} C_{j+1, l, m} \\
& D_{j, k, m}^{h}=\sum_{l, n} h_{l-2 k} g_{n-2 m} C_{j+1,1, n}
\end{aligned}
$$

A medical CT image of sports tear injure is decomposed into a low frequency component and a series of high frequency components in different directions after the wavelet multiscale analysis (Eq. 1). The low frequency component maintains its original content information. A series of highfrequency components represent significant details for the medical CT image of sports tear injure, such as edges and regional boundaries [11]. In the above equation, $A_{j, k, m}$ represents the low frequency coefficient for medical CT images of sports tear injure. $D_{j, k, m}^{h}$, and $D_{j, k, m}^{d}$ represent coefficients of the high-frequency edge information for medical CT images of sports tear injury in the horizontal, vertical, and diagonal directions, respectively. $h_{l-2 k}$ is the coefficient of the low pass filter. $g_{n-2 m}$ is the coefficient of the high pass filter. $C_{j+1, n}$ represents a multi-scale wavelet decomposition vector.

\subsubsection{Multi-directional image enhancement algorithm based on wavelet layering}

For medical CT images of sports tear injure after wavelet decomposition, image enhancement should be performed on components in different directions [12]. Low frequency part is an approximation for medical CT images of sports tear injure. It is a relatively smooth part for medical CT images of sports tear injure. Gray value changes are relatively flat and do not have unidirectionality [13]. Enhancement of the low frequency part is mainly to adjust gray scale range and improve quality for medical CT images of sports tear injure. Therefore, it is enhanced by wavelet-based homomorphic filtering. Enhancement of the high frequency portion is an important part of the entire image. High frequency component contains detail features and noise of the image and has three separate 
unidirectional directions: horizontal, vertical and diagonal. Therefore, in this paper, high-frequency components in the three directions of the image are denoised first, and then weight enhancement processing of the three components is performed [14].

Enhancement of low frequency information Low-frequency part for medical CT image of sports tear injury is enhanced. It mainly adjusts grayscale range of $\mathrm{CT}$ images to enhance their image contrast. According to the actual situation, lowfrequency part is enhanced by wavelet-based homomorphic filtering [15]. Wavelet-based homomorphic filtering enhancement is applied to traditional homomorphic filtering enhancement based on wavelet multi-scale analysis. As shown in Fig. 2, WT represents an n-level wavelet transform, IWT represents a corresponding inverse wavelet transform, and LF represents a Butterworth homomorphic filter. $f(x, y)$ represents an original medical CT image of sports tear injury, and $g(x, y)$ represents a processed medical CT image of sports tear injury. In this paper, wavelet-based homomorphic filtering algorithm is used to enhance the low-frequency components of medical images. Image contrast is enhanced while adjusting the overall gray scale for medical image of sports tear injure. Further expanding gray scale of the image, the processed CT image of sports tear injure better maintains original appearance [16].

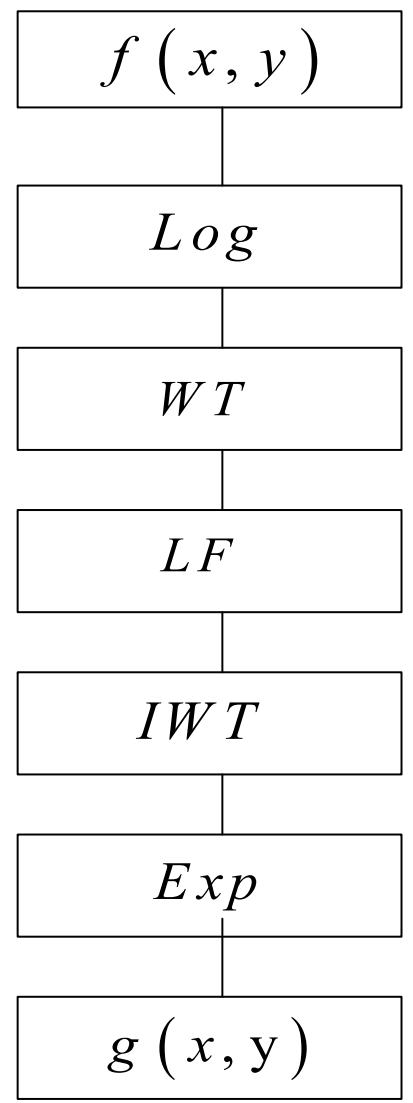

Fig. 2 Homomorphic Enhancement Based on Wavelet
The Butterworth homomorphic filter function (Butterworth filter) is used [17]. Its main principle structure is represented in Fig. 3.

Specific design of the homomorphic filter conversion function based on wavelet analysis is as follows:

$H_{A(x, y)}=\left(\gamma_{1}-\gamma_{2}\right)\left\{k \times\left[A_{j, k, m}(x, y)-m\right]+m\right\}$,

where $H_{A(x, y)}$ is a wavelet-based homomorphic filtering enhancement transfer function that enhances low-frequency components of the image. The expression $k \times\left[A_{j, k, m}(x, y)-\right.$ $m]+m$ is a linear equalization denoising of low frequency components. $\gamma_{1} \gamma_{2}$ is the correction factor, $A_{j, k, m}(x, y)$ is the wavelet coefficient of low-frequency component for medical CT image of sports tear injure. $m$ is the average value for wavelet coefficient of the low-frequency region. $\mathrm{k}$ is a contrast adjustment factor for adjusting the unevenness of image brightness. Choosing appropriate parameter $\mathrm{k}$ maintains the original appearance of medical CT images while enhancing local contrast of the image. Enhancement processing of low frequency component is implemented such that the low frequency component $H_{A} \leq 1$. Dynamic range of the compressed image as a whole is achieved while enhancing the contrast between adjacent parts of the image, therefore, $0 \leq k \leq 1$ is selected.

Enhancement of high frequency information Detailed features and noise for medical CT images of sports tear injure are mainly distributed in the high frequency region. High frequency component has three different directions: horizontal, vertical, and diagonal. If wavelet coefficients in different directions of the high frequency part are directly denoised, some important details of the image may be lost. Threshold denoising enhancement processing is performed on images in different directions by wavelet sparseness to better preserve the detailed features of medical CT images. It is divided into two steps: firstly, denoising multi-directional wavelet coefficients in the high-frequency components of the image, and then performing weighting enhancement on the denoised wavelet function.

\section{Step 1: Denoising}

Because imaging process for medical CT images of sports tear injure is susceptible to noise interference, its imaging process is expressed as:

$g(i, j)=f(i, j)+n(i, j)$,

where $i=1,2, \ldots, M, j=1,2, \ldots, N, g(i, j)$ represents a medical $\mathrm{CT}$ image of sports tear injury contaminated by noise. $f(i, j)$ is a clear image, that is, a real image without noise. $n(i, j)$ is image-independent noise. In medical CT images of sports tear injure, according to the central limit 
Fig. 3 Principle structure of Butterworth Homomorphism filter function

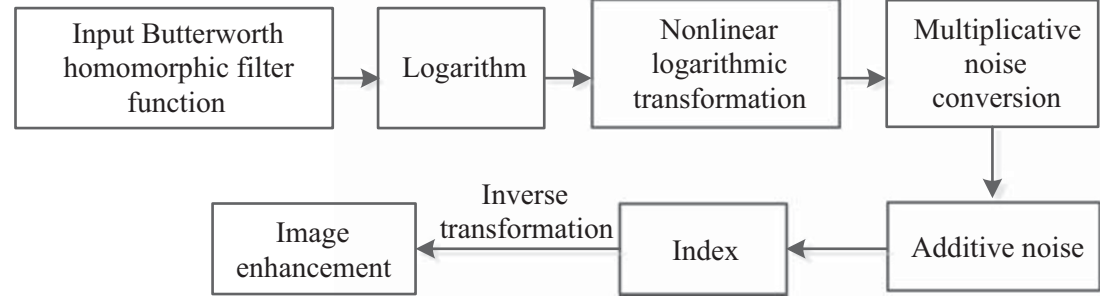

theorem, $n(i, j)$ is generally a Gaussian white noise with a mean of zero.

For the degraded image $g(i, j)$ of size $M \times N$, the wavelet second decomposition $W_{\phi}\left(k_{0}, x, y\right)$ and the reconstruction $W_{y}^{l}$ $(k, x, y)$ are expressed as:

$W_{\phi}\left(k_{0}, x, y\right)=\frac{1}{\sqrt{M N}} \sum_{i=0}^{M-1} \sum_{j=0}^{N-1} g(i, j) \phi_{k 0, x, y}(i, j) C_{j+1,1, n}$

$W_{y}^{l}(k, x, y)=\frac{1}{\sqrt{M N}} \sum_{i=0}^{M-1} \sum_{j=0}^{N-1} g(i . j) y_{k, x, y}^{l}(i, j) \quad l=\{1,2,3\}$

where $\phi_{k 0, x, y}(i, j), y_{k, x, y}^{l}(i, j)$ are translation basis functions. $k_{0}$ is an arbitrary starting scale, usually taking $k_{0}=1$ and choosing $M=N=2^{K}, k-1, x 、 y \in\left\{0,1, \cdots, 2^{k}-1\right\} . M, N$ and $x, y$ represent adjustment factors.

After wavelet transformation, medical CT images and noise from sports tear injure will show different statistical properties [18]. Energy for medical CT image signals of sports tear injure is more concentrated on the wavelet coefficient and the low-resolution coefficient. But the noise energy is distributed on different scales. Relatively speaking, high frequency part is more concentrated. In other words, spatial correlation for CT images of sports tear injure in the wavelet domain is reduced. Energy is concentrated, but energy distribution of the noise is unchanged. Thus, the wavelet coefficients are divided into two categories. One is the result of image detail transformations that contain noise. This part of the wavelet coefficient has a larger amplitude, but the number is smaller. The other type is the wavelet coefficient caused by noise. This part of the coefficient is a large number, but its amplitude is relatively small. Therefore, wavelet coefficients are processed by means of the threshold. A wavelet coefficient greater than the threshold is considered to be a useful signal to be reserved, while a wavelet coefficient less than the threshold is considered to be rejected. The threshold $T$ generally used is:

$T=\sigma \sqrt{2 \ln (N)}$,

where $N$ is the total number of pixels for medical CT images of sports tear injury, $\sigma=$ median $\left(\left|w_{x y}\right| / 0.6745\right), w_{x y}$ is the wavelet coefficient of the highest frequency subband, and $\sigma$ indicates spatial correlation.

For medical CT images of sports tear injure, edge detail features often contain important disease characteristics. Therefore, how to preserve the image detail features while removing noise is necessary for medical CT images of sports tear injure [19].

\section{Step 2: Weighting enhancement of multi-directional wavelet coefficients after denoising}

Wavelet coefficients in different directions in the highfrequency components for medical $\mathrm{CT}$ images of sports tear injure are denoised. It is considered that the processed wavelet coefficients are basically important features such as details and edges in the image [20]. The wavelet coefficients in different directions after denoising are subjected to weight enhancement processing. Specifically, wavelet coefficients of different directions in the high-frequency component are respectively introduced with different weights $K_{j, k, m}$ (acquired by Eq. (9)), so as to achieve important features such as image detail enhancement to different degrees.

$f(x, y)=\sum_{k, m} A_{j, k, m} \phi(x, y)+\sum_{i=0} \sum_{j=0} K_{j, k, m} D_{j, k, m}^{c} \phi(x, y)$,

where $K_{j, k, m}$ indicates enhancement degree of the image detail after denoising. High frequency components in medical CT images of sports tear injure are further enhanced. Details of the image are relatively prominent. The processed medical CT image of sports tear injure has an overall contour enhancement and improved sharpness. Enhancement effect is more obvious.

Multi-scale image enhancement The original medical CT image of sports tear injure is decomposed by wavelet. Corresponding wavelet coefficients are obtained at each scale. They respectively represent an approximate portion for a medical CT image of sports tear injure, a detail portion having high frequency information in the horizontal direction, vertical direction, and diagonal direction. Wavelet coefficients in different directions of each scale are enhanced by the abovementioned enhancement algorithm to complete multi-scale enhancement for medical CT images of sports tear injure.

\subsubsection{Image edge extraction algorithm based on extended mathematical morphology}

Mathematical morphology edge detection operator needs to select the appropriate structural elements. According to the basic principles of morphology such as expansion, corrosion, morphological opening operations, morphological closing 
operations, and their combined operations, clear image edges are obtained [21]. Selection of structural elements determines effectiveness and accuracy of the processing results. For edge extraction of $\mathrm{CT}$ images, appropriate structural elements must be selected according to texture structure of the image. Moreover, the size, shape and orientation of structural elements must be fully considered. Usually, the square structure elements of $3 \times 3$ or $5 \times 5$ is selected, and different structural elements are selected according to requirements and experience.

According to the final result relationship of morphological operation, corrosion and expansion for the enhanced medical $\mathrm{CT}$ image of sports tear injury is defined as:

$f(x, y) \Theta B \subseteq f(x, y) \subseteq f(x, y) \oplus B$,

where $\Theta$ and $\oplus$ are arithmetic symbols. $f(x, y)$ represents an enhanced medical CT grayscale image of sports tear injury. $B$ represents the grayscale structural element for the enhanced medical CT image of sports tear injury.

Morphological opening and morphological closing operations satisfy:

$f(x, y)^{\circ} B \subseteq f(x, y) \subseteq f(x, y) \cdot B$,

where $f(x, y)^{\circ} B$ represents an open operation in morphology and $f(x, y) \cdot B$ represents a closed operation in morphology.

Edge extraction process of $\mathrm{CT}$ images based on extended mathematical morphology for sports tear injury is:

Let $E_{d}(F)$ denote a dilation residue edge detector. It is defined as the difference between the enhanced medical CT image $f(x, y)$ by the expansion of structural element $B$ and the original image:

$E_{d}(F)=(f(x, y) \oplus B)-f(x, y)$

Similarly, let $E_{e}(F)$ denote an erosion residue edge detector. It is defined as the difference between the original image and the image $f(x, y)$ that has been etched by structural element $B$ as:

$E_{e}(F)=f(x, y)-(f(x, y) \Theta B)$

Let $G(F)$ denote morphological gradient of the image $f(x$, $y$ ). It is obtained by expansion and corrosion operations:

$G(F)=R(F)(f(x, y) \oplus B)-(f(x, y) \Theta B)$,

where $M=\left(f(x, y)^{\circ} B\right)$ indicates an open operation. $B$ is a square structural element of $3 \times 3$.

Preprocessing is performed by morphological opening operation and closing operation to filter out noise. Then do the morphological opening operation to smooth the image. Finally do the expansion operation. The difference between the image after expansion and the image before expansion obtains a better image edge. $R(F)$ is the image edge extraction result for medical CT images of sports tear injure.

\subsection{CT image feature extraction algorithm based on NSCT-GLCM}

\subsubsection{Non-downsampled contour transformation}

Non-downsampled contour transformation (NSCT) includes a pyramid filter bank (NSPFB) and a directional filter bank (NSDFB). Its structural principle is shown in Fig. 4. NSCT segmentation process is described as follows. Multi-scale segmentation for medical $\mathrm{CT}$ images of sports tear injury is first performed by NSCT based on the extracted edge $R(F)$. High frequency sub-band images and a low frequency sub-band image of various frequencies are obtained. Resulting high frequency sub-band images are then multi-directionally transformed using NSDFB. Thus sub-band images of different scales and directions are obtained [22].

\subsubsection{Gray level co-occurrence matrix}

Linear distance is $\mathrm{d}$ from one pixel point where the gray value for medical CT images of sports tear injure is $i$. The gray value of another pixel at angle $\theta$ is $j$. Probability $p(i, j, d, \theta)$ of two gray values occurring simultaneously in the entire medical CT image of sports tear injure is:

$p(i, j, d, \theta)=\{(x, y) \mid f(x, y)=i, f(x+d \cos \theta, y+d \sin \theta)=j\}$,

where $x, y$ represents medical $\mathrm{CT}$ image factors of sports tear injure. Commonly used texture features for medical CT images of sports tear injure are homogeneous region (HOM),

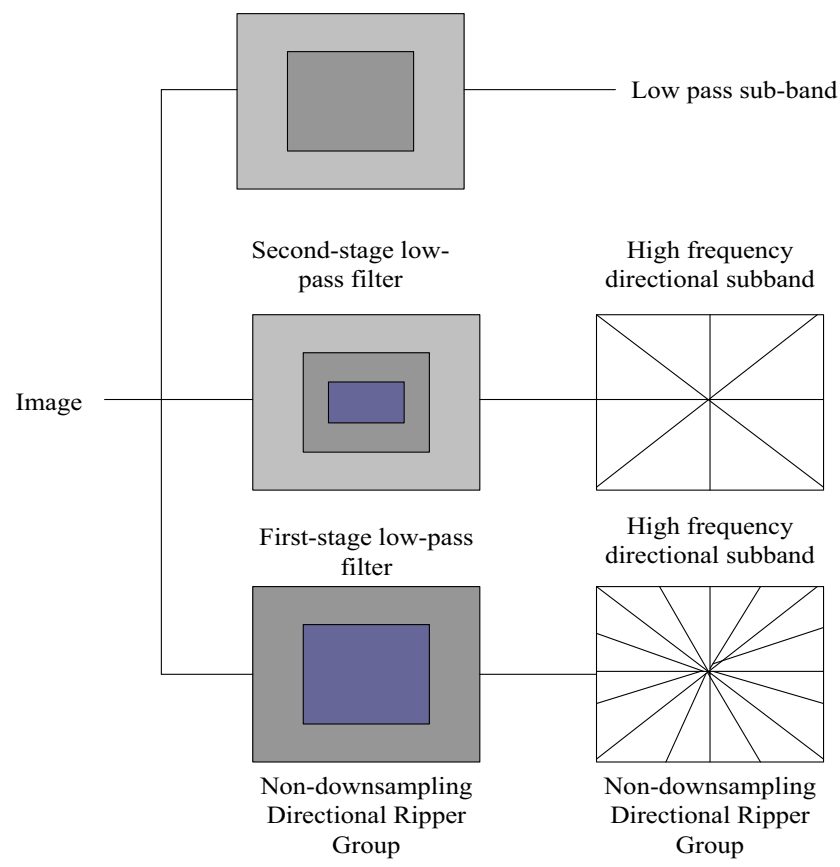

Fig. 4 Principle of NSCT 
angular second moment (ASM), entropy (ENT), and dissimilarity (DIS). They are defined as follows:

$$
\begin{aligned}
& H O M=\sum_{i} \sum_{j} p(i, j) /\left[1+(i-j)^{2}\right] \\
& A S M=\sum_{i} \sum_{j}[p(i, j)]^{2} \\
& E N T=\sum_{i} \sum_{j} p(i, j) \lg (p(i, j) \\
& D I S=\sum_{i} \sum_{j}|i-j| p(i, j)
\end{aligned}
$$

\subsubsection{Extracting image texture features using NSCT-GLMC}

Let the input medical CT image of sports tear injure be $f(x, y)$, and its size is $M \times N$. Process of NSCT segmentation is expressed as:

$f(x, y)=a_{J}+\sum_{j=1}^{n} \sum_{k=1}^{m} b_{j, k}$,

where $b_{j, k}$ is the high-frequency sub-band for CT image of sports tear injure in the $j$-scale and $k$-direction. $a_{J}$ is the lowfrequency sub-band for medical CT image of sports tear injury. Feature extraction steps based on NSCT-GLCM are as follows:

\section{(1) NSCT segmentation for medical CT images of sports tear injure}

An image is first transformed into two layers by NSCT based on the extracted edge $R(F)$. The number of first dimension directions is 8 [23], and the number of second scale directions is 4 . Thus, a medical CT image is divided into sub-bands having different scales and different directions. A total of 13 sub-bands of the same size as the original image are obtained. Each direction subband represents a texture in one direction.

\section{(2) Quantification of transform coefficients}

NSCT low-frequency sub-band $a_{J}$ approximates a uniform distribution. High-frequency sub-band for medical CT image of sports tear injury shows a distribution of "high peaks and long tails". Low-frequency sub-band for medical CT image of sports tear injury is uniformly quantized. The quantization level is 16 , which is quantized according to Eq. (21) as:

$G=\left[\left(C-C_{\min }\right) /\left(C_{\max }-C_{\min }\right)\right] \times 16$,

where $G$ denotes a quantization result, $C$ denotes a quantization vector, $C_{\min }$ denotes a minimum value of quantization vector, and $C_{\max }$ denotes a maximum value of quantization vector. Non-uniform quantization for CT image detail subband of medical tear injury is used. The coefficients are quantized to 16 levels. First estimate variance $\sigma_{l}$ of each sub-band at the same scale. When quantifying, $\pm 3 \sigma$ is used as the limit and segmentation is quantized according to Eq. (22) as:

$$
G_{l}=\left\{\begin{array}{l}
{\left[\left(C-C_{\min }\right) /\left(C_{\max }-C_{\min }\right)\right] \times 12,|C(m, n)| \leq 3 \sigma_{l}} \\
{\left[\left(C-C_{\min }\right) /\left(C_{\max }-C_{\min }\right)\right] \times 2,|C(m, n)|>3 \sigma_{l}}
\end{array}\right.
$$

(3) Window selection, including window traversal mode and window size

GLCM's window traversal image mode uses overlapping windows. Different sizes of windows are used at different NSCT segmentation scales. Sub-band image of the first scale for medical CT image of sports tear injure use $N=3 \times 3$ size window. The second-scale sub-band image and the lowfrequency sub-band use a window of $N=5 \times 5$ size.

\section{(4) Distance parameter}

With setting of NSCT segmentation parameters, the number of segmentation directions will change. Differences between the scales will vary with the change of direction parameters. Therefore, when NSCT split sub-band is used for gray level symbiosis, different distance intervals $d$ are used for different scales.

\section{(5) Selection of direction}

Symbiotic amount is extracted by GLCM Eqs. 19 to 22 . Four symbiotic quantities of HOM, ASM, ENT, and DIS are calculated for each subband of NSCT at four angles (angles $\theta$ are $0^{\circ}, 45^{\circ}, 90^{\circ}$, and $135^{\circ}$, respectively). The values of four different directions for each symbiotic amount are then averaged as shown in Eq. (23). In this way, robustness of gray level symbiosis to direction is improved.

$f=\left(f^{0}+f^{45}+f^{90}+f^{135}\right) / 4$

Finally, 52-dimensional symbiotic feature $N_{-} G(m, n)$ of point $(m, n)$ for medical CT image of sports tear injury is obtained. CT image feature extraction of sports tear injury is completed.

$N_{-} G(m, n)=\left[H_{O M}, A M S_{i}, E N T_{i}, D I S_{i}\right]$

\section{Results and analysis}

In order to verify effectiveness of the proposed algorithm, a simulation experiment is needed. Matlab is selected as the 
experimental platform, and the algorithm in this paper is programmed in matlab, and the methods in literature $[1,5]$ are used as experiments for this method, and the algorithms are programmed and run on the Matlab platform. In the experiment, a total of $1000 \mathrm{CT}$ images were selected for training and feature selection in the 3D-IRCADb 01 database (https:// www.ircad.fr/research/3d-ircadb-01/), in order to test the effectiveness of the algorithm in this paper. Ready. Experimental software and hardware parameter settings are shown in Tables 1 and 2:

According to the above experimental parameter settings, performance of the proposed algorithm is analyzed.

\subsection{Analysis of effectiveness}

In order to analyze effectiveness of the proposed algorithm, the algorithm is applied to an actual CT image feature extraction experiment of sports tear injury. Figure 5 is a raw medical $\mathrm{CT}$ image of sports tear injury. Figure 6 is a feature image proposed by the algorithm.

Analysis of Figs. 5 and 6 shows that features for CT image of sports tear injure extracted by the algorithm are clear. Texture features of injured part of the leg are better extracted.

\subsection{Analysis of performance}

In order to accurately analyze performance advantages of the proposed algorithm, Literature [1] algorithm, Literature [5] algorithm and the proposed algorithm are compared. Under normal conditions, when medical CT images of sports tear injure are being imaged, machine noise interferes with the quality of the image. Therefore, denoising, enhancement and extraction time consumption of CT images by three algorithms are compared. The experimental results are as follows:

\section{(1) Denoising}

Gaussian noise is introduced into medical CT image of sports tear injury, as shown in Fig. 7. The comparison results for denoising of three algorithms are shown in Figs. 8, 9 and 10.

Analysis of Figs. 7, 8, 9 and 10 shows that there are a large number of noise points in medical CT image of sports tear injure in which Gaussian noise is introduced. In the CT image after denoising, noise points are almost invisible. Leg details of sports tear injury are clearer. Noise points for medical CT image of sports tear injure after denoising by Literature [1]

Table 1 Hardware environment

\begin{tabular}{lll}
\hline Hardware & CPU & RAM \\
\hline Server & $\geqq 2.2 \mathrm{HZ}$ & $\geqq 1024 \mathrm{M}$ \\
Client & $\geqq 1.6 \mathrm{HZ}$ & $\geqq 512 \mathrm{MB}$ \\
\hline
\end{tabular}

Table 2 Software environment

\begin{tabular}{ll}
\hline Software & Type \\
\hline Operating System & Windows 2015/IIS6.0 \\
Development Tools & Power Builder 9.0 \\
$\quad$ Database & SQL Server 2013 \\
\hline
\end{tabular}

algorithm are removed. However, horizontal stripes left by denoising appear in the image, which affects sharpness of the CT image. Literature [5] algorithm has a poor effect on the removal of noise points in medical CT images of sports tear injury. The processed CT image still has some noise points, and denoising effect is lower than the proposed algorithm. It can be seen that denoising performance of the proposed algorithm is significant.

\section{(2) Enhancement}

Original CT image of sports tear injure from scanning devices may be degraded due to various factors such as imaging device and acquisition conditions, as shown in Fig. 11. Three algorithms are used for image enhancement processing. The contrast enhancement results are shown in Figs. 12, 13 and 14.

Analysis of Figs. 11, 12, 13 and 14 shows that medical CT image of sports tear injure with degraded image quality is relatively vague, which is not conducive to judgment of diseases. Image quality enhanced by the algorithm is obviously improved. It can be seen that the enhanced image has clear details, strong layering and higher resolution. Medical CT image of sports tear injure enhanced by Literature [1] algorithm and Literature [5] algorithm is overexposed. Local edge is unclear and local contrast enhancement effect is not

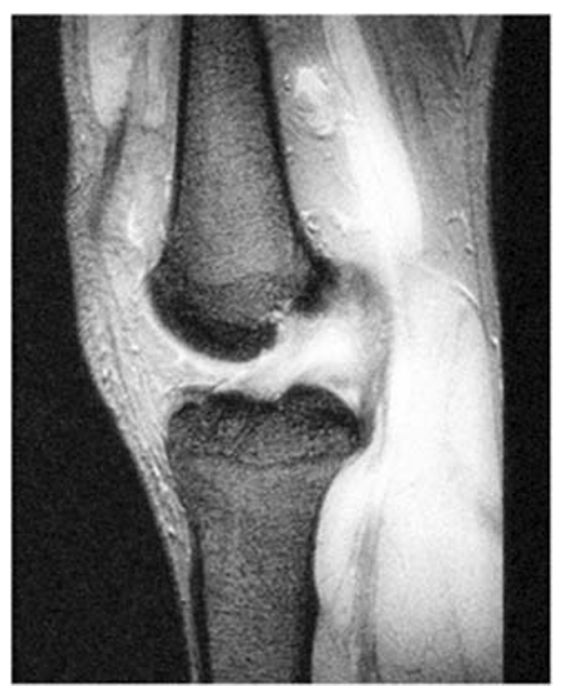

Fig. 5 Medical CT image of original sports tear injury 


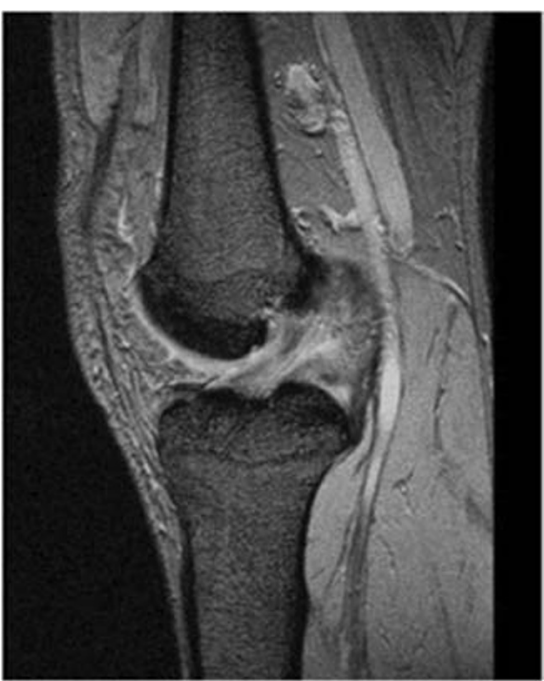

Fig. 6 The feature extraction results of the algorithm in this paper

obvious. It shows that the algorithm has the best enhancement effect.

\section{(3) Analysis of extraction efficiency}

In medical CT image analysis, diagnostic efficiency of a condition depends not only on records of other examination reports, but also on analysis efficiency of CT images. Therefore, feature extraction efficiency for medical CT images of sports tear injure is set as a comparison index of three algorithms. A total of 7 patients with sports tear injury are set to examine the condition by medical CT imaging. Comparing time-consuming data of three algorithms in extracting the futures for medical CT images of sports tear injure. The comparison results are shown in Fig. 15:

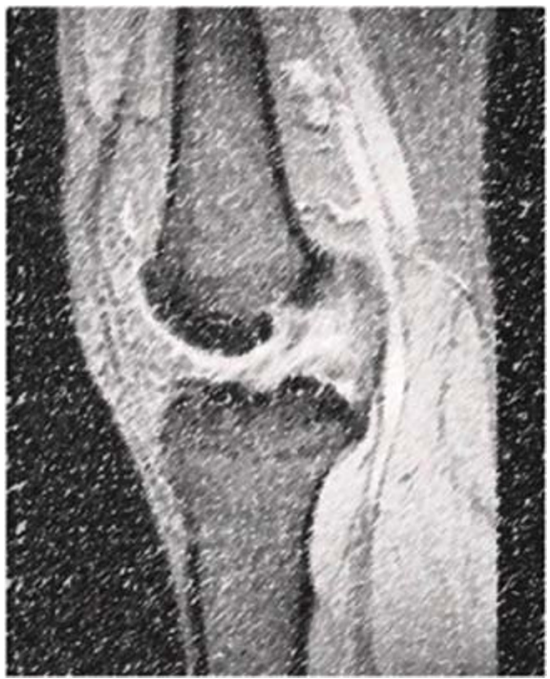

Fig. 7 Medical CT images of sports tear injury imported with Gauss noise

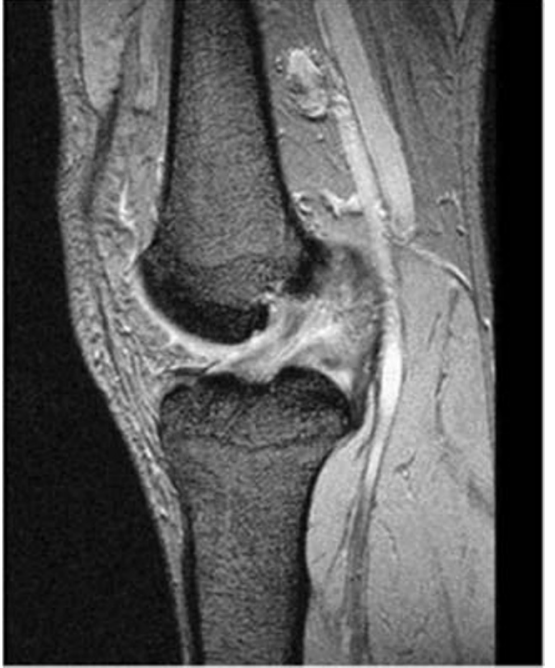

Fig. 8 Medical CT image of sports tear injury after denoising by this algorithm

Analysis of Fig. 15 shows that extraction time of the algorithm is stable at $2 \mathrm{~ms}$ when extracting the features for 7 medical CT image of sports tear injure. Literature [5] algorithm takes $21 \mathrm{~ms}$ to extract $\mathrm{CT}$ image features of the first patient, and feature extraction time for $\mathrm{CT}$ images of remaining 6 patients is $30 \mathrm{~ms}$. Extraction time of Literature [1] algorithm is $45 \mathrm{~ms}$. By comparison, extraction algorithm in this paper takes the shortest time.

\section{Conclusion}

Medical image visualization is an important research content of scientific computing visualization. It is an important application of computer graphics and image processing in biomed-

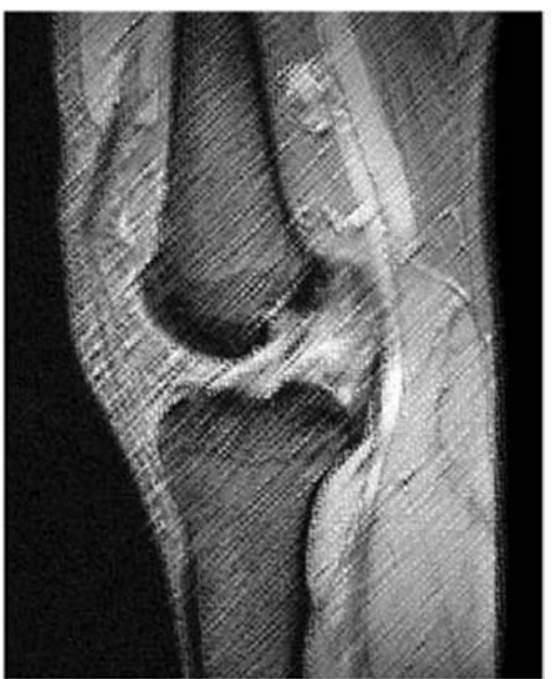

Fig. 9 Medical CT images of sports tear injury after denoising by Literature [1] algorithm 


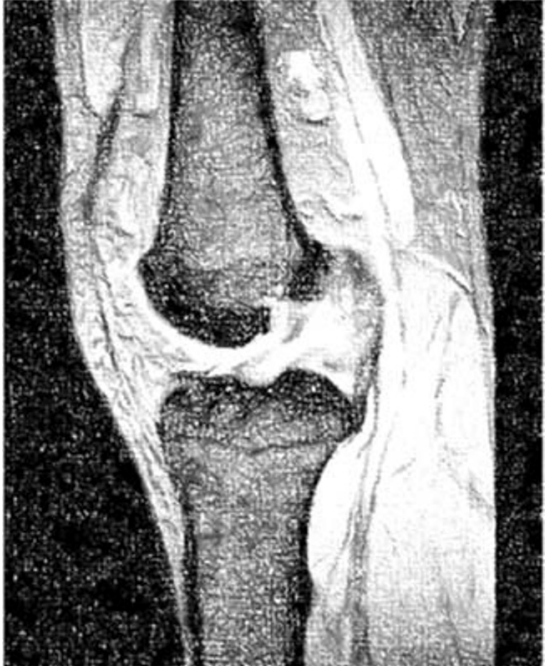

Fig. 10 Medical CT image of sports tear injury after denoising by Literature [5] algorithm

ical engineering. It uses computer graphics and image processing techniques to reconstruct medical 2D image data into 3D solid images. It helps doctors to understand internal tissue information of human body and analyze diseased body with its surrounding tissues. Accuracy and scientificity of medical diagnosis are improved. It is conducive to development of optimal treatment plans and radiological planning. Moreover, it is of great significance in clinical, educational and medical research. Today's macroscopic CT has been able to directly observe organ lesions, which is equivalent to the pathological structure seen by pathological autopsy. Structure of tissues and cells is observed by Micro-CT, without taking biopsy, without positioning puncture, without using case slices. It directly makes clinical case diagnosis in real time

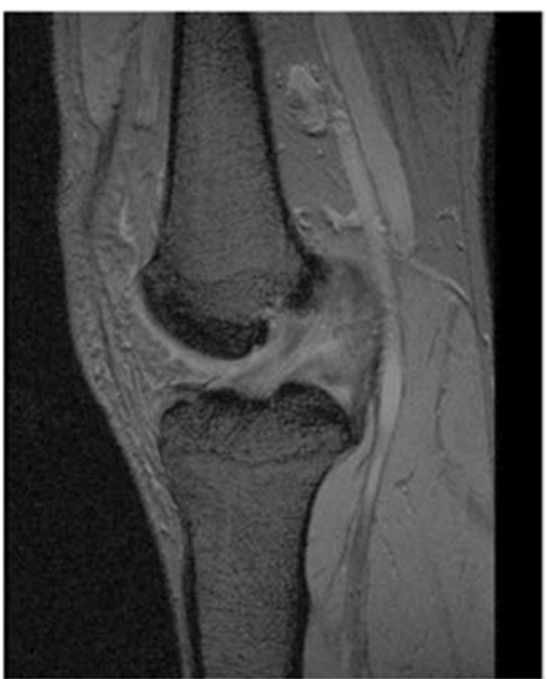

Fig. 11 Medical CT images of sports tear injury with image quality degradation

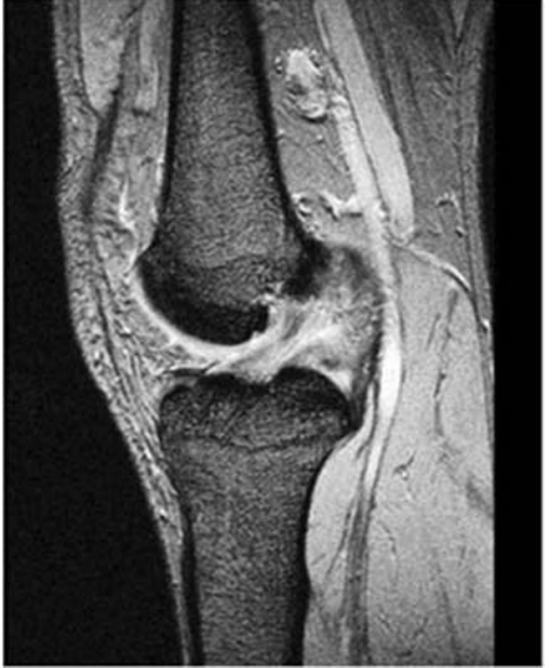

Fig. 12 Medical CT images of sports tear injury enhanced by our algorithm

without non-invasive. Considering that medical raw $\mathrm{CT}$ images are affected by various factors such as imaging equipment and acquisition conditions, as well as machine noise, degradation of image quality and blurring of edges may occur.

A feature extraction algorithm for medical CT images of sports tear injury is proposed. First, medical CT image of sports tear injure is preprocessed. After CT image of sports tear injure is analyzed by wavelet multi-scale, the image is decomposed into a low frequency component and a series of high frequency components in different directions. Then, multi-directional image enhancement algorithm based on wavelet layering is used to enhance the low-frequency information and high-frequency information of the image, so as to achieve multi-scale enhancement for $\mathrm{CT}$ images of sports tear

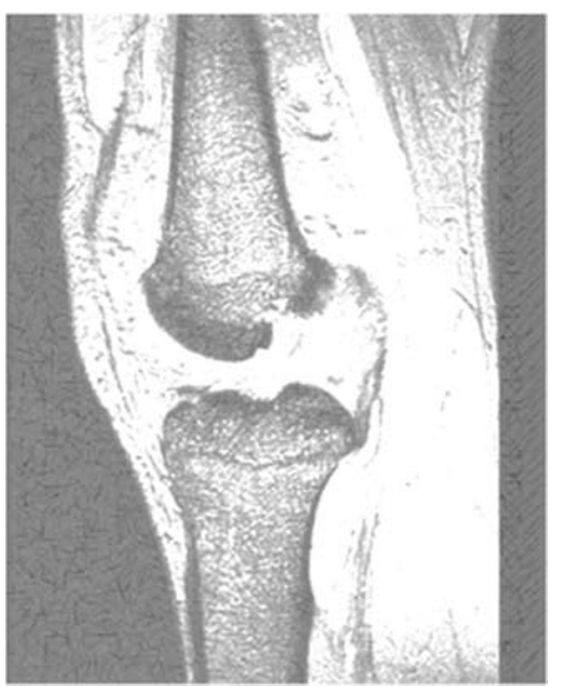

Fig. 13 Medical CT images of sports tear injury enhanced by Literature [1] algorithm 


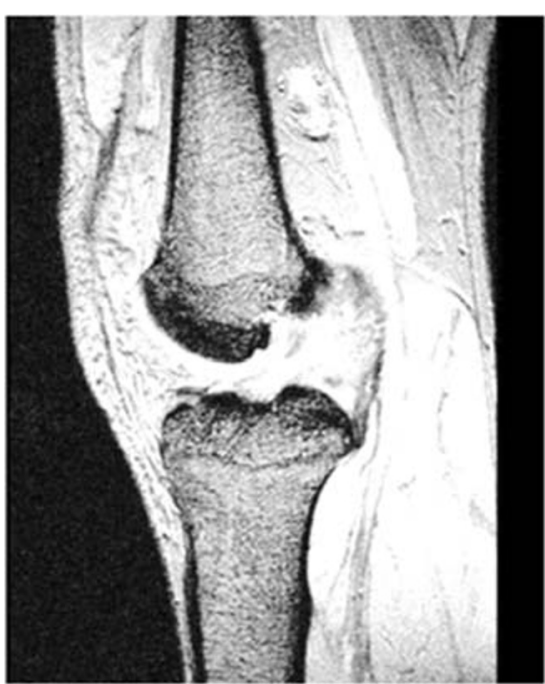

Fig. 14 Medical CT images of sports tear injury enhanced by Literature [5] algorithm

injure. The proposed algorithm preserves important features of the image while removing noise from the image. Finally, image edge extraction algorithm based on extended mathematical morphology is used to extract edge of the enhanced $\mathrm{CT}$ image. According to the extracted edge information, CT image feature extraction algorithm based on NSCT-GLCM is used to complete feature extraction for CT images of sports tear injury. The algorithm effectively eliminates pixel missegment in regions and obtain more accurate CT image segmentation results.

Experiments show that the proposed algorithm effectively extracts CT image features of sports tear injury. The image with noise interference has strong denoising performance. In addition, medical CT image of sports tear injure enhanced by the proposed algorithm has clear details, strong layering and higher resolution.

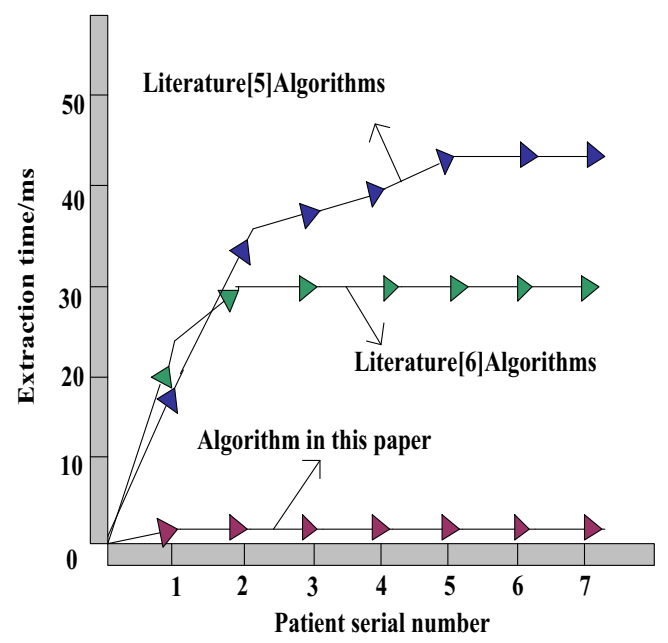

Fig. 15 Time-consuming comparison results of three algorithms
Acknowledgements This paper is supported by the project "Application of driven Teaching in PHYSICAL Education curriculum" with No.JXJG17-12-9 and the project "Research on the Anti-Exercise Fatigue Effect and Mechanism of Jiawei Shengmai Powder".

Funding Open Access funding provided by Western Norway University Of Applied Sciences.

\section{Compliance with ethical standards}

Ethics information "Feature extraction for medical CT images of sports tear" The research content of the project strictly abides by the international ethical standards for biomedical research involving human beings formulated by the World Health Organization and the Council of international medical science organizations, as well as the relevant provisions of the National Natural Science Foundation of China. In the implementation process of this project, we will strictly do a good job in informed consent, inform the source of the use of biological samples and treatment scheme, ensure that the personal information and medical information of the sample source are not publicly disclosed, and make every effort to protect the privacy of the personal medical data, disease information and life information of the sample source within the scope of law.

Open Access This article is licensed under a Creative Commons Attribution 4.0 International License, which permits use, sharing, adaptation, distribution and reproduction in any medium or format, as long as you give appropriate credit to the original author(s) and the source, provide a link to the Creative Commons licence, and indicate if changes were made. The images or other third party material in this article are included in the article's Creative Commons licence, unless indicated otherwise in a credit line to the material. If material is not included in the article's Creative Commons licence and your intended use is not permitted by statutory regulation or exceeds the permitted use, you will need to obtain permission directly from the copyright holder. To view a copy of this licence, visit http://creativecommons.org/licenses/by/4.0/.

\section{References}

1. Ema T, Yamagata H, Aoyagi K (2017) Medical image processing apparatus, ultrasound imaging apparatus, X-ray CT apparatus. Field Crop Res 63(3):189-197

2. Zeng R, Gavrielides M, Petrick N (2016) Estimating local noise power spectrum from a few FBP-reconstructed CT scans. Med Phys 43(1):568-582

3. Liu J, Hoffman J, Zhao J (2016) Mediastinal lymph node detection and station mapping on chest CT using spatial priors and random forest. Med Phys 43(7):4362-4374

4. Hu Y, Liang Z, Song B (2016) Texture feature extraction and analysis for polyp differentiation via computed tomography colonography. IEEE Trans Med Imaging 35(6):1522-1531

5. Shaukat F, Raja G, Gooya A (2017) Fully automatic detection of lung nodules in CT images using a hybrid featureset. Med Phys 44(7):3615-3629

6. Wollenweber SD, Kemp BJ (2016) Technical note: rod phantom analysis for comparison of PET detector sampling and reconstruction methods. Med Phys 43(11):6175-6185

7. Huang KW, Lin CC, Lee YM, Wu ZX (2019) A deep learning and image recognition system for image recognition. Data Sci Patt Recog 3(2):1-11

8. Shuai L, Zheng P, Xiaochun C (2017) A novel fast fractal image compression method based on distance clustering in high dimensional sphere surface. Fractals 25(4):1740004 
9. Veeraraghavan H (2016) MO-A-207B-01: Radiomics: Segmentation \& Feature Extraction Techniques. Med Phys 43(6): 3694-3694

10. Reddy GT, Reddy MP, Lakshmanna K, Kaluri R, Rajput DS, Srivastava G, Baker T (2020) Analysis of dimensionality reduction techniques on big data. IEEE Access 16(8):54776-54788

11. Tam A, Barker J, Rubin D (2016) A method for normalizing pathology images to improve feature extraction for quantitative pathology. Med Phys 43(1):528-537

12. Shuai L, Wang S, Xinyu L et al (2020) Fuzzy Detection aided Realtime and Robust Visual Tracking under Complex Environments. IEEE Trans Fuzzy Syst. https://doi.org/10.1109/TFUZZ.2020. 3006520

13. Gastounioti A, Oustimov A, Keller B (2016) Breast parenchymal patterns in processed versus raw digital mammograms: a large population study toward assessing differences in quantitative measures across image representations. Med Phys 43(11):5862-5877

14. Liu S, Bai W, Liu G et al (2018) Parallel fractal compression method for big video data. Complexity 2018:2016976

15. Liu S, Liu G, Zhou H (2019) A robust parallel object tracking method for illumination variations. Mobile Netw Appl 24(1):5-17

16. Vu T, Mousavi H, Monga V (2016) Histopathological image classification using discriminative feature-oriented dictionary learning. IEEE Trans Med Imaging 35(3):738-751

17. Al-Sahaf H, Zhang M, Al-Sahaf A (2017) Keypoints detection and feature extraction: a dynamic genetic programming approach for evolving rotation-invariant texture image descriptors. IEEE Trans Evol Comput 21(6):825-844

18. Shuai L, Xinyu L, Shuai W et al (2020) Fuzzy-aided solution for out-of-view challenge in visual tracking under IoT assisted complex environment. Neural Comput \& Applic. https://doi.org/10.1007/ s00521-020-05021-3

19. Adamo T, Ghiani G, Grieco A (2017) MIP neighborhood synthesis through semantic feature extraction and automatic algorithm configuration. Comput Oper Res 83(C):106-119

20. Chang H, Lee M, Lee W (2016) Feature extraction-based Hellinger distance algorithm for nonintrusive aging load identification in residential buildings. IEEE Trans Ind Appl 52(3):2031-2039

21. Long Z, Qian G, Dong X (2017) K- local maximum margin feature extraction algorithm for churn prediction in telecom. Clust Comput 20(2):1401-1409

22. Shuai L, Chunli G, Fadi A et al (2020) Reliability of response region: a novel mechanism in visual tracking by edge computing for IIoT environments. Mech Syst Signal Process 138:106537

23. Shahdoosti H, Javaheri N (2018) A fast algorithm for feature extraction of hyperspectral images using the first order statistics. Multimed Tools Appl 77(4):1-18

Publisher's note Springer Nature remains neutral with regard to jurisdictional claims in published maps and institutional affiliations. 